\title{
SIMULATION-BASED PERFORMANCE ANALYSIS OF AUTOMATED SINGLE-TRAY VERTICAL LIFT MODULE
}

\author{
Rosi, B. ; Grasic, L. ; Dukic, G. ${ }^{* *}$; Opetuk, T. ${ }^{* *} \&$ Lerher, T. \\ *University of Maribor, Faculty of Logistics, Mariborska cesta 7, 3000 Celje, Slovenia \\ ${ }^{* *}$ University of Zagreb, Faculty of Mechanical Engineering and Naval Architecture, \\ Ivana Lučića 5, 10002 Zagreb, Croatia \\ E-Mail: bojan.rosi@um.si, leon.grasic@ mors.si,goran.dukic@fsb.hr, tihomir.opetuk@fsb.hr, \\ tone.lerher@uni-mb.si
}

\begin{abstract}
In this paper a simulation analysis of an automated single-tray Vertical Lift Module (VLM) system is presented. Compared to other well-known warehouse systems, a VLM system can provide moderate throughput capacity and lower investment cost. The objective of the study is to investigate benefits of a single-tray VLM system design in terms of reducing transactions' mean cycle time, which results in increased throughput of the system. Performance of the single-tray VLM is evaluated regarding alternative design configurations and the velocity profiles.

The results show that single-tray VLM systems are effective and may serve as guideline for warehouse designers in designing a new or improving an existing automated warehouse system.

(Received in May 2015, accepted in October 2015. This paper was with the authors 2 months for 2 revisions.)
\end{abstract}

Key Words: Logistics, Automated Warehouse, Warehousing, Vertical Lift Module, Discrete Event Simulation, Performance Analysis

\section{INTRODUCTION}

Warehouses are logistics systems. Based on a traditional saying, a system is a complex or very complex feature made of a set of elements and a set of these elements' relations. Due to relations and hence interactions, a system is more than a sum; the whole system has properties, which single elements do not have: it has synergies [1]. Systems science is required by the complexity of modern technology's "systems", which is also the case for logistics system in modern warehouses [2].

There are many reasons why warehouses are required, like facilitation of the coordination between the supply and demand using buffers of products, accumulation and consolidation of products from several producers for combined shipments, enabling same-day delivery service for customers, supporting product customization activities like packaging or assembly, etc. [3, 4].

Typically, a warehouse system consists of several sub-systems (areas), which are related with each other. At the receiving area, incoming shipments with products are unloaded from trucks or railway, and inspected whether quantity and quality of the products are as ordered. Products could be transported directly to the shipping area, which is also known as a "crossdocking" process, or could be transported and placed to the "reserve and forward" area and later on the shipping area. At the shipping area, outgoing shipments with products are checked for the quality and quantity, issued to the internal costumer or loaded to trucks or trains for distribution to the external customers [3-5].

Automated systems play significant role in warehouses, studied by a lot of researchers over the past few decades. Intensive developments of automated systems have begun with the development of computer science and information technology, being an important and almost inevitable part of today's modern warehouses. Main advantages of automated warehousing are savings in labour costs and used floor space, increased accuracy (reduced error rates) and 
high reliability. The most frequently used automated systems are systems to store and retrieve unit-load and mini-load items without interference of an operator, named Automated Storage and Retrieval Systems (AS/RS). AS/RS have quite long history of the application in warehouses, since first installations appeared in 1960s.

Due to a large product variety accompanied with the customer's requirements for fast deliveries (sometimes at the same day) and more frequent deliveries (many smaller orders), material handling system producers are constantly developing new solutions of automated storage systems. To meet throughput demand of small parts and layout related constraints, automated Vertical Lift Modules (VLM), have been developed (Fig. 1).
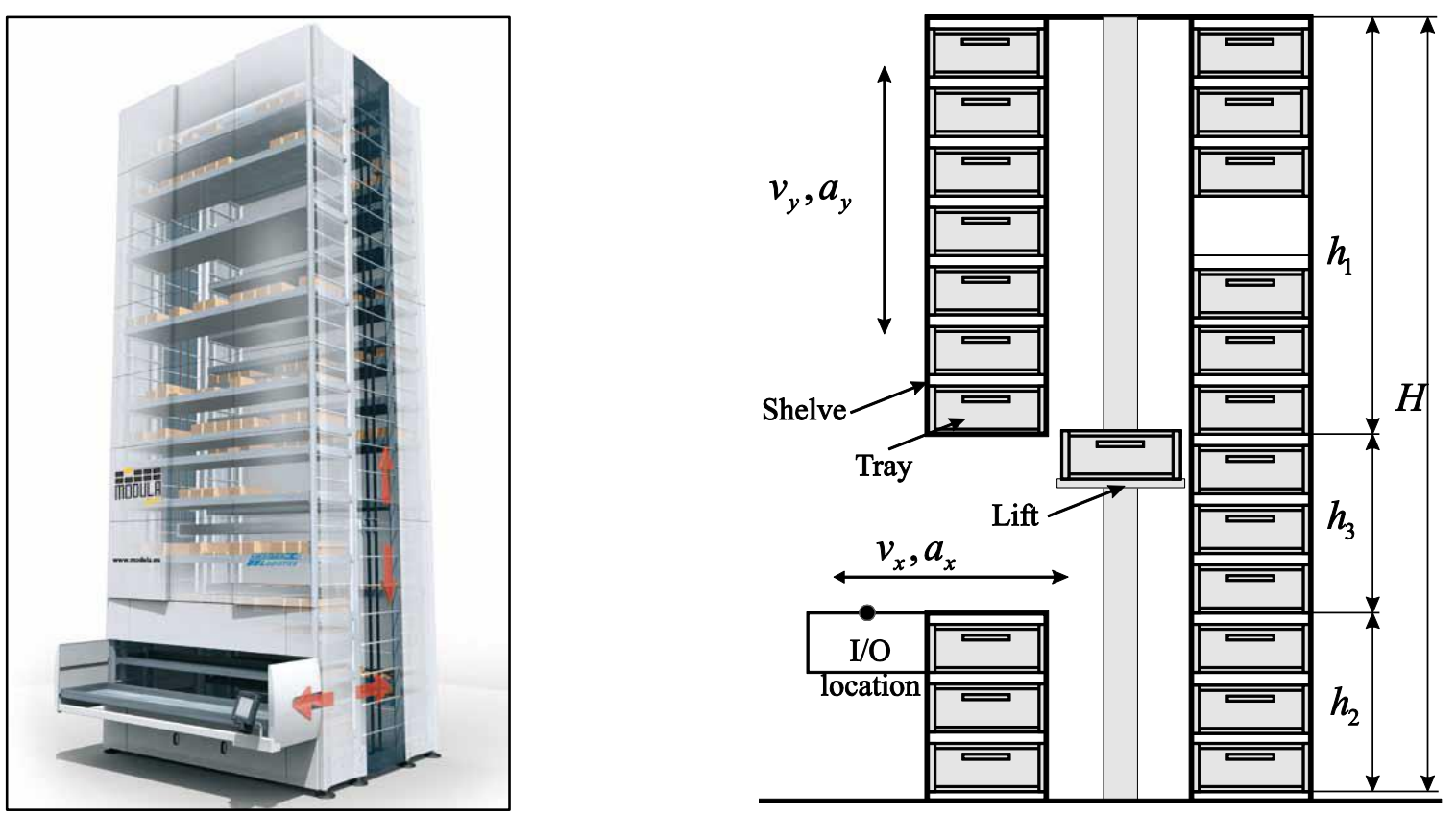

Figure 1: Vertical lift module of the material handling provider MODULA (http://www.modula.eu/).

This new automated system, VLM, has been developed as an alternative to picker-to part order picking system with shelving. In this technology, the insertion/extraction device called as a lift is capable of vertical movement of trays with products (Fig. 1), thus delivering parts to the picker.

The goal of this paper is an evaluation of VLM's throughput performance by using discrete event simulation technique. The VLM performance is evaluated based on system's throughput which depends on the expected system's cycle time. The rest of the paper is structured as follows. Literature review is given in Section 2. Section 3 presents simulation model of the VLM system after the brief description of VLM device, while section 4 presents analysed VLM design scenarios. Simulation results with relevant discussion are presented in Section 5. Main conclusions from this study are given in final section 6.

\section{LITERATURE REVIEW}

AS/RS are studied well in the literature. There are primarily two types of AS/RS: unit-load AS/RS and the mini-load AS/RS. AS/RS usually consist of conveyors, storage racks and automated Storage/Retrieval (S/R) machines travelling in narrow aisles between the storage racks to store and retrieve loads. The $\mathrm{S} / \mathrm{R}$ machine can manipulate either pallets (unit-load system) or totes (mini-load system). Many studies of AS/RS have been performed in the material handling research community in the past few decades, from which only some are mentioned bellow. 
Hausman et al. [6] and Graves et al. [7] were among the first presenting travel time models for AS/RS, also analysing different storage strategies, e.g. randomized, turnoverbased and class-based storage assignment rules, however only for so called square-in-time racks (SIT rack). Gudehus [8] presented principles for calculation of the cycle time for the Single Command (SC) cycle and Dual Command (DC) cycle. In case of the SC cycle, the S/R machine can perform storage or retrieval request per cycle. More advanced is the DC cycle where both storage and the retrieval request are done in one cycle. With regard to other cycle time models, he also considered the influence of the acceleration and deceleration on calculated cycle time. Bozer and White [9] presented analytical travel time models of SC and DC cycles for non-SIT racks. Models are based on randomized storage and retrieval request, assuming that the S/R machine travels all the time with constant velocity. Hwang and Lee [10] presented travel time models considering the real operating characteristics of the $S / R$ machine in non-SIT racks. Sari et al. [11] presented closed-form travel-time expressions for flow-rack AS/RS. Lerher et al. [12] developed analytical travel time models for multi-aisle AS/RS considering the operating characteristics of the S/R machine. With the proposed analytical travel time models average travel time can be evaluated. Gu et al. [13] presented a comprehensive review of research on warehouse operation. Roodbergen and Vis [14] presented an extensive overview of the literature on AS/RS. Lerher et al. [15] presented simulation analysis of mini-load multi-shuttle AS/RS while Lerher et al. [16] presented simulation analysis of Shuttle Based Storage and Retrieval Systems (SBS/RS).

Smew et al. [17] presented a simulation study about production and inventory control at the supply chain level, examining the Hybrid Kanban-CONWIP production control strategy. Bekker [18] proposed the extended buffer allocation problem (BAP) to the multi objective method and proposed a method to optimise two conflicting objectives of the BAP, namely throughput performance and allocation buffer capacity. Berlec et al. [19] proposed the methodology for the calculation of the optimal batch quantity by using the basic and the extended model, which consider the tied-up capital in a production along to the cost of changing the batch and storage cost.

Erkan and Can [20] used AHP (Analytic Hierarchy Process) and FAHP (Fuzzy Analytic Hierarchy Process) to decide on Barcode or RFID system to select for a company, in order to collect data for its warehouse. As a result of the study, it is found that AHP produces consistent results with FAHP. Barcode system is selected for the data collection system and FAHP is found to be more sufficient in decision making process due to its fuzziness and vagueness compared to AHP. Seebacher et al. [21] presented an applicable approach (timebased efficiency concept) of the intralogistics efficiency. For the evaluation of intralogistics process they used discrete event simulation modelling technique. Karabegovic at al. [22] presented the usefulness and the application of service robots named by automatic guided vehicles (AGVs) into intralogistics process. This technology offers high efficiency, high scalability, high operation availability $(98.5 \%)$, etc. On the other hand these systems are rather expensive; therefore they need to be carefully planned into intralogistics process.

Systems like carousels (horizontal and vertical) and VLM received much less attention in literature than the others. The most important paper that presented throughput models for carousels and VLM was the paper from Meller and Klote [23]. The VLM assumed in this paper was with single picking place (so called single-tray or single-level delivery VLM). With the appearance of VLM with two pick places on opening (so called dual-tray or dual-level delivery), a throughput model for such systems was proposed in the paper of Dukic et al. [24].

Other papers regarding VLM are more like technical papers focusing on application areas and benefits of using VLM compared to a shelving system, plenty of them being published by producers. 
Unlike the (mentioned) existing studies, in this paper we approach the single-tray VLM design from a velocity profile design of lift by using discrete event simulation modelling technique.

\section{SIMULATION MODEL OF VLM}

VLM are used in warehouses and industrial applications since the early 1970's. A VLM can be viewed as enclosed, six-sided box that works using three columns. The front and the rear columns are functioning like shelves to store trays and totes. The centre column is used for lift, which operates within front and rear columns in vertical motion to store or retrieve trays by order [24].

First versions were much slower and with limited capability in storage and retrieval orders. Today's VLM provide increased operational velocity, higher weight capacities, automated control systems and easy-to-use user interface. The operator (order picker) requests a tray or SKU number via keypad or touch-screen display. The lift is directed to the exact position to extract a tray from storage location and to deliver it at the pick window. Operator picks products and presses button to confirm completion. The tray is then returned to its storage location. The process can be repeated depending on the size of the order or a batch of orders [24].

In the simulation model of VLM the following assumptions and notations were used.

\section{Main assumptions}

- VLM is divided into front and the rear columns, equipped with brackets to support trays with products (Fig. 1).

- The centre column is used for the lift, which operates within front and rear columns in vertical motion to store or retrieve trays by order (Fig. 1).

- When a tray is requested by an operator, it is extracted and delivered to the work station window, where the $\mathrm{I} / \mathrm{O}$ location is set.

- The lift moves trays up and down to and from the I/O location. The horizontal movement of trays is illustrated with picking up and depositing times.

- The lift moves one tray at a time. There is one pick place for delivered trays.

- The lift complete retrieval commands only.

- Drive characteristics $\left(v_{y}, a_{y}\right)$ of the lift as well as the heights $h_{1}, h_{2}, h_{3}$ and $H$ of the VLM are known in advance.

- Randomized assignment policy has been considered.

\section{Notations}

$\begin{array}{ll}\text { AHP } & \text { Analytic Hierarchy Process. } \\ \text { AS/RS } & \text { Automated storage and retrieval systems. } \\ \text { AGV } & \text { Automatic guided vehicles. } \\ \text { DC } & \text { Dual command. } \\ \text { FAHP } & \text { Fuzzy Analytic Hierarchy Process. } \\ \text { I/O } & \text { Input and output. } \\ \text { I/E } & \text { Insertion/extraction device. } \\ \text { RFID } & \text { Radio frequency identification. } \\ \text { SC } & \text { Single command. } \\ \text { S/R } & \text { Storage and retrieval. } \\ \text { SIT } & \text { Square in time. } \\ \text { vp } & \text { Velocity profile. } \\ \text { VLM } & \text { Vertical lift module. } \\ a_{y} & \text { Acceleration/deceleration of the lift in the vertical direction. }\end{array}$




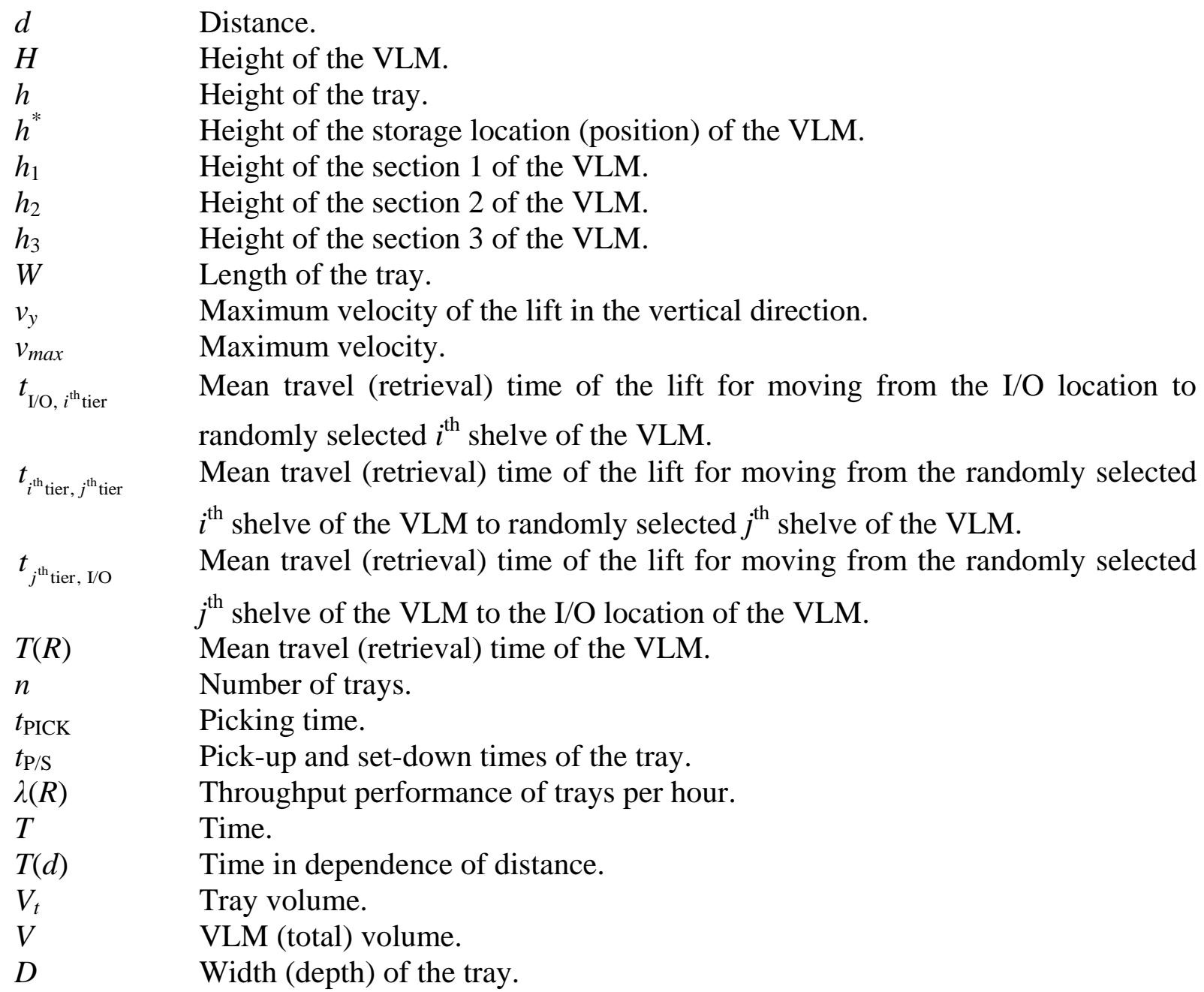

Discrete event simulation was used to evaluate the performance of the VLM system. Movement of the lift is based on the real velocity-time dependence, of which two types can be distinguished. If the obtained peak velocity $v\left(t_{p}\right)$ is less than $v_{\max }$ we named it type I, while if the obtained peak velocity $v\left(t_{p}\right)$ is equal to $v_{\max }$ we named it type II.

Lift movement for type $\mathbf{I} .\left(d<v^{2} / 2\right)$

Time in dependence of distance $T(d)$ is calculated by (1)

$$
T(d)=2 \sqrt{\frac{d}{a}}
$$

Lift movement for type II. $\left(d \geq v^{2} / 2\right)$

Time in dependence of distance $T(d)$ is calculated by (2)

$$
T(d)=\frac{d}{v}+\frac{v}{a}
$$

In our paper retrieval requests for the parts picking have been analysed only (therefore refilling storage containers was not considered). In the case of a VLM, retrieval operations correspond with dual command cycles. This means that after the order picker (operator) picks all the products from the tray at the I/O location, the lift moves back the tray to the $i^{\text {th }}$ shelve of the VLM. Next, the lift takes another tray from the $j^{\text {th }}$ shelve of the VLM and moves back to the I/O location. Selection of trays in the $i^{\text {th }}$ and the $j^{\text {th }}$ shelve of the VLM is based on randomly selected location, which corresponds with the random storage strategy. 
According to the retrieval request selection rule in VLM, mean travel (retrieval) time $T(R)$ of lift equals collection of (Fig. 2):

- mean travel (retrieval) time from the I/O location of the VLM to randomly selected $i^{\text {th }}$ shelve of the VLM;

- mean travel (retrieval) time from randomly selected $i^{\text {th }}$ shelve of the VLM to randomly selected $j^{\text {th }}$ shelve of the VLM;

- mean travel (retrieval) time from randomly selected $j^{\text {th }}$ shelve of the VLM to the I/O location of the VLM.
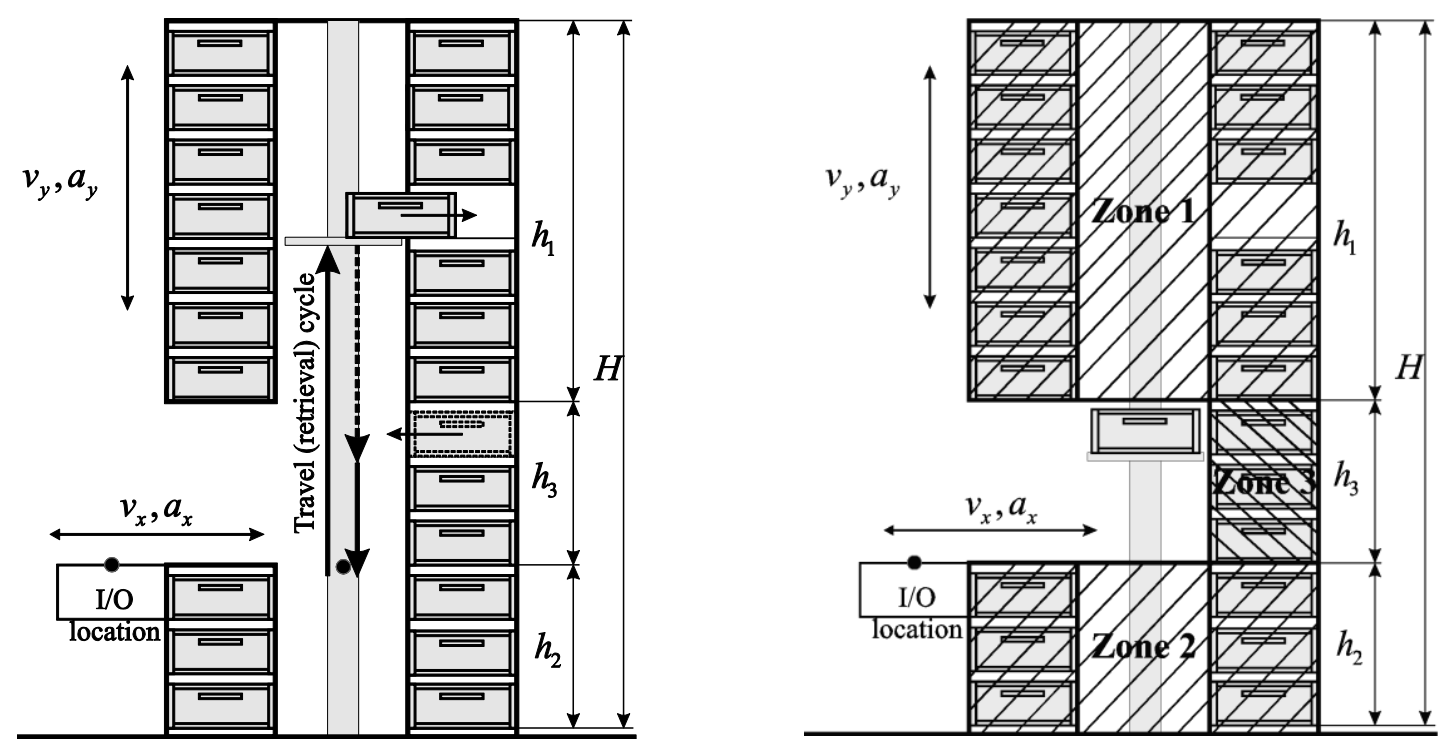

Figure 2: Definition of the travel (retrieval) cycle and zoning of the VLM.

Since the VLM is divided into three areas or zones (Fig. 2), probabilities $p_{i}$ for accessing zones different zones are calculated by (3) [24]:

$$
p_{1}=\frac{2 h_{1}}{\left(2 H-h_{3}\right)} ; p_{2}=\frac{2 h_{2}}{\left(2 H-h_{3}\right)} ; p_{3}=\frac{h_{3}}{\left(2 H-h_{3}\right)} ;
$$

Mean cycle (retrieval) time $T(R)$ consists of mean lift's travel times, additional times for pick-up and set-down and picking time (including identification and confirmation) and equals (4):

$$
T(R)=t_{\mathrm{PICK}}+4 \cdot t_{\mathrm{P} / \mathrm{S}}+t_{\mathrm{I} / \mathrm{O}, i^{\mathrm{th}}} \text { tier }+t_{i^{\mathrm{th}}}{ }_{\text {tier }, j^{\mathrm{th}}}{ }_{\text {tier }}+t_{j^{\mathrm{th}}}{ }_{\text {tier }, \mathrm{I} / \mathrm{O}}
$$

By considering (4) the throughput performance known as number of trays per hour is calculated by (5):

$$
\lambda(R)=\frac{3600}{T(R)}
$$

\section{VLM CASE STUDY}

This section presents and discusses main input data for the analysis. Total volume $V$ of all spare parts is set to $10 \mathrm{~m}^{3}$. Trays filled with items (storage containers) have the following dimensions: length of the tray $W=1.9 \mathrm{~m}, 2.5 \mathrm{~m}, 3.1 \mathrm{~m}$ and $4.1 \mathrm{~m}$, width (depth) of the tray $D=0.857 \mathrm{~m}$ and height of the tray $h=0.12 \mathrm{~m}$. Height of the section $1 h_{1}$ of the VLM is a variable value, which has an influence on the tray capacity $n_{1}$ of the section 1 (Fig. 2). Height of the section $2 h_{2}$ of the VLM was set to $0.6 \mathrm{~m}$ with tray capacity $n_{2}=6$ trays (Fig. 2). Height of the section $3 h_{3}$ of the VLM was set to $0.6 \mathrm{~m}$ with tray capacity $n_{3}=3$ trays (Fig. 2). Note that the height of the storage location is higher than the tray and is marked with $h^{*}=0.2 \mathrm{~m}$. 
Dimensions of the VLM ( $W, D$ and $H$ ) depend on the selected tray dimensions and number of trays. Velocity scenarios of the lift are as follows: $\left(\mathrm{vp}_{1}\right)$ stands for $v_{y}=1.0 \mathrm{~m} / \mathrm{s}$, $a_{y}{ }^{+}=a_{y}{ }^{-}=0.5 \mathrm{~m} / \mathrm{s}^{2} ;\left(\mathrm{vp}_{2}\right)$ stands for $v_{y}=1.0 \mathrm{~m} / \mathrm{s}, a_{y}{ }^{+}=a_{y}{ }^{-}=1.5 \mathrm{~m} / \mathrm{s}^{2} ;\left(\mathrm{vp}_{3}\right)$ stands for $v_{y}=1.5 \mathrm{~m} / \mathrm{s}, a_{y}{ }^{+}=a_{y}{ }^{-}=1.5 \mathrm{~m} / \mathrm{s}^{2} ;\left(\mathrm{vp}_{4}\right)$ stands for $v_{y}=2.0 \mathrm{~m} / \mathrm{s}, a_{y}{ }^{+}=a_{y}{ }^{-}=1.5 \mathrm{~m} / \mathrm{s}^{2}$ and $\left(\mathrm{vp}_{5}\right)$ stands for $v_{y}=2.0 \mathrm{~m} / \mathrm{s}, a_{y}{ }^{+}=a_{y}{ }^{-}=2.0 \mathrm{~m} / \mathrm{s}^{2}$. Pick-up and delivery time $t_{P / D}$ is set to 4.0 seconds. Velocity scenarios from $\mathrm{vp}_{1}$ to $\mathrm{vp}_{5}$ were chosen based on the material handling equipment producers' references and authors' practical experiences.

As seen in Table I, four $\mathrm{VLM}_{i}$ configurations are analysed. Geometrical data ( $W, D, h$ and $h^{*}$ ) were used from the material handling provider Modula (http://www.modula.eu/).

Table I: VLM configurations.

\begin{tabular}{|c|c|c|c|c|}
\hline $\begin{array}{c}\text { VLM } \\
\text { configurations }\end{array}$ & $\mathbf{V L M}_{\mathbf{1}}$ & $\mathbf{V L M}_{\mathbf{2}}$ & $\mathbf{V L M}_{\mathbf{3}}$ & $\mathbf{V L M}_{\mathbf{4}}$ \\
\hline$W(\mathrm{~m})$ & 1.9 & 2.5 & 3.1 & 4.1 \\
\hline$D(\mathrm{~m})$ & 0.857 & 0.857 & 0.857 & 0.857 \\
\hline$h(\mathrm{~m})$ & 0.12 & 0.12 & 0.12 & 0.12 \\
\hline$h^{*}(\mathrm{~m})$ & 0.20 & 0.20 & 0.20 & 0.20 \\
\hline$V_{t}\left(\mathrm{~m}^{3}\right)$ & 0.20 & 0.25 & 0.32 & 0.42 \\
\hline$h_{1}(\mathrm{~m})$ & 4.2 & 3.2 & 2.4 & 1.6 \\
\hline$n_{1}(/)$ & 42 & 32 & 24 & 16 \\
\hline$h_{2}(\mathrm{~m})$ & 0.6 & 0.6 & 0.6 & 0.6 \\
\hline$n_{2}(/)$ & 6 & 6 & 6 & 6 \\
\hline$h_{3}(\mathrm{~m})$ & 0.6 & 0.6 & 0.6 & 0.6 \\
\hline$n_{3}(/)$ & 3 & 3 & 3 & 3 \\
\hline$H(\mathrm{~m})$ & 5.4 & 4.4 & 3.6 & 2.8 \\
\hline$n(/)$ & 51 & 41 & 33 & 25 \\
\hline$p_{1}(/)$ & 82 & 78 & 73 & 64 \\
\hline$p_{2}(/)$ & 12 & 15 & 18 & 24 \\
\hline$p_{3}(/)$ & 6 & 7 & 9 & 12 \\
\hline
\end{tabular}

Volume of the single tray is calculated by (6):

$$
V_{t}=W \cdot D \cdot h
$$

Number of trays according to the total volume $V$ is calculated by (7):

$$
n=\frac{V}{V_{t}}
$$

Heights of the VLM sections are calculated by (8):

$$
h=n-9 \text { trays }
$$

Height of the rack (VLM) is calculated by (9):

$$
H=h_{1}+h_{2}+h_{3}
$$

\section{ANALYSIS OF RESULTS AND DISCUSSION}

Table II summarizes mean cycle (retrieval) time of the lift $T(R)$ for four (4) different VLM configurations, which were analysed according to five (5) different velocity profiles of the lift (see Chapter 4). 
Table II: Mean cycle (retrieval) time $T(R)$ of the VLM.

\begin{tabular}{|c|c|c|c|c|}
\hline \multirow{2}{*}{$\begin{array}{c}\text { Velocity } \\
\text { profile }\end{array}$} & \multicolumn{4}{|c|}{$\boldsymbol{T}(\boldsymbol{R})$} \\
\cline { 2 - 5 } & $\mathrm{VLM}_{1}$ & $\mathrm{VLM}_{2}$ & $\mathrm{VLM}_{3}$ & $\mathrm{VLM}_{4}$ \\
\hline $\mathrm{vp}_{1}$ & 27.7 & 26.1 & 24.9 & 23.6 \\
\hline $\mathrm{vp}_{2}$ & 25.1 & 23.7 & 22.6 & 21.5 \\
\hline $\mathrm{vp}_{3}$ & 23.0 & 22.0 & 21.2 & 20.4 \\
\hline $\mathrm{vp}_{4}$ & 22.6 & 21.8 & 21.1 & 20.4 \\
\hline $\mathrm{vp}_{5}$ & 21.9 & 21.1 & 20.5 & 19.8 \\
\hline
\end{tabular}

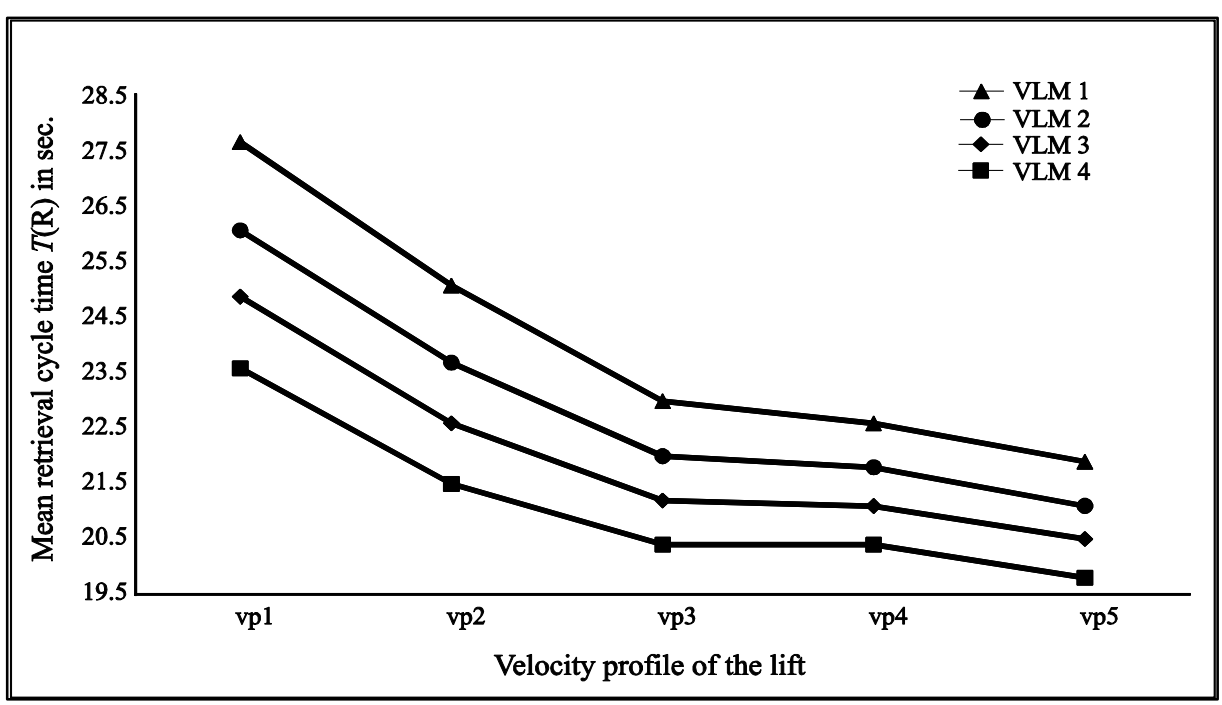

Figure 3: VLM cycle (retrieval) time analysis.

Mean cycle (retrieval) time $T(R)$ of the lift depends on the height $H$ of the VLM and the velocity profile $\operatorname{vp}_{i}\left(v_{y}\right.$ and $\left.a_{y}\right)$ of the lift. The fastest transactions of the lift belong to VLM with relatively small height $H\left(\mathrm{VLM}_{4}\right)$. The above-mentioned condition stands for all velocity profiles $\mathrm{vp}_{i}$ of the lift (Fig. 3). On the contrary, the slowest transactions of the lift belong to VLM with relatively large height $H\left(\mathrm{VLM}_{1}\right)$.

Regarding to the distribution of the mean cycle (retrieval) time $T(R)$, an expressive dependency has been noticed (Fig. 3) between the velocity profile $v_{i}$ and the mean cycle (retrieval) time $T(R)$. In general, the best results are achieved by the lift with fast drives in the vertical travelling direction (Fig. 3).

Table III summarizes VLM performance measured in number of trays per hour for different VLM configurations (see Chapter 4).

Table III: Number of trays per hour of the VLM $_{i}$.

\begin{tabular}{|c|c|c|c|c|c|c|c|c|c|c|c|c|}
\hline \multirow{4}{*}{$\begin{array}{c}\text { Velocity } \\
\text { profile }\end{array}$} & \multicolumn{12}{|c|}{$\lambda(\boldsymbol{R})$} \\
\hline & \multicolumn{3}{|c|}{$\mathrm{VLM}_{1}$} & \multicolumn{3}{|c|}{$\mathrm{VLM}_{2}$} & \multicolumn{3}{|c|}{$\mathrm{VLM}_{3}$} & \multicolumn{3}{|c|}{$\mathrm{VLM}_{4}$} \\
\hline & \multicolumn{3}{|c|}{ Picking time } & \multicolumn{3}{|c|}{ Picking time } & \multicolumn{3}{|c|}{ Picking time } & \multicolumn{3}{|c|}{ Picking time } \\
\hline & 5 & 15 & 30 & 5 & 15 & 30 & 5 & 15 & 30 & 5 & 15 & 30 \\
\hline $\mathrm{vp}_{1}$ & 110 & 84 & 62 & 116 & 88 & 64 & 120 & 90 & 66 & 126 & 93 & 67 \\
\hline $\mathrm{vp}_{2}$ & 120 & 90 & 65 & 125 & 93 & 67 & 130 & 96 & 68 & 136 & 99 & 70 \\
\hline $\mathrm{vp}_{3}$ & 129 & 95 & 68 & 133 & 97 & 69 & 137 & 99 & 70 & 142 & 102 & 71 \\
\hline $\mathrm{vp}_{4}$ & 130 & 96 & 68 & 134 & 98 & 70 & 138 & 100 & 70 & 142 & 102 & 71 \\
\hline $\mathrm{vp}_{5}$ & 134 & 98 & 69 & 138 & 100 & 70 & 141 & 101 & 71 & 145 & 103 & 72 \\
\hline
\end{tabular}




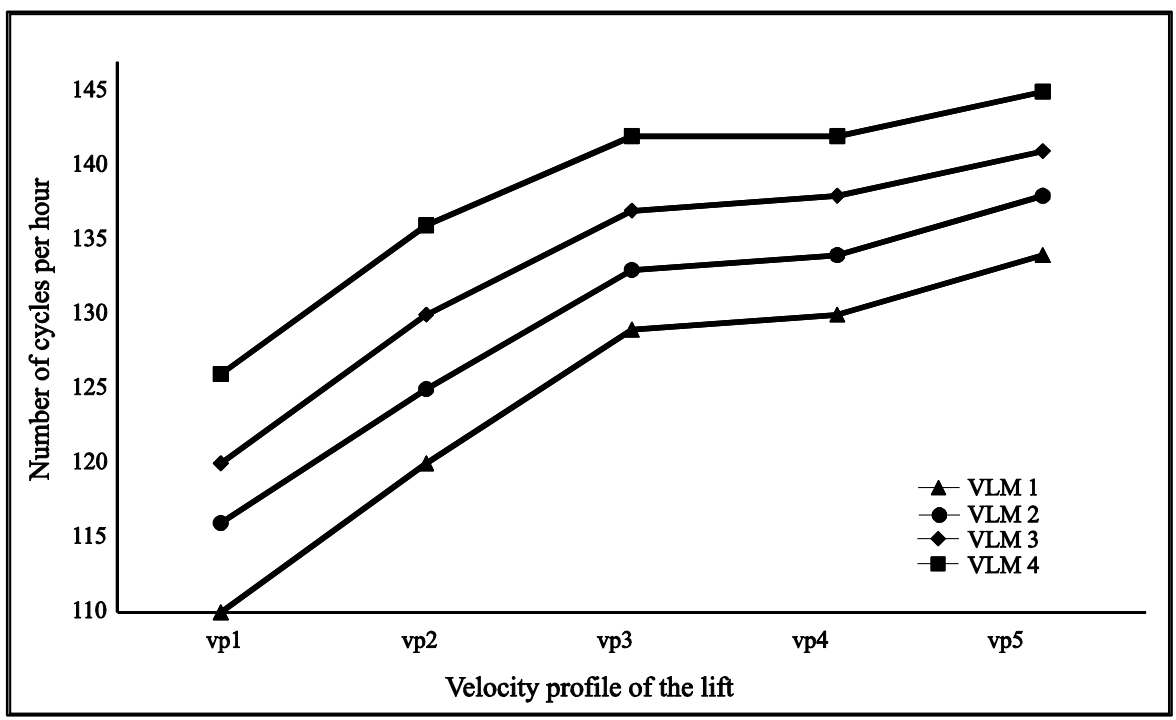

Figure 4: VLM performance in number of trays per hour when $t_{\text {pick }}=5 \mathrm{sec}$.

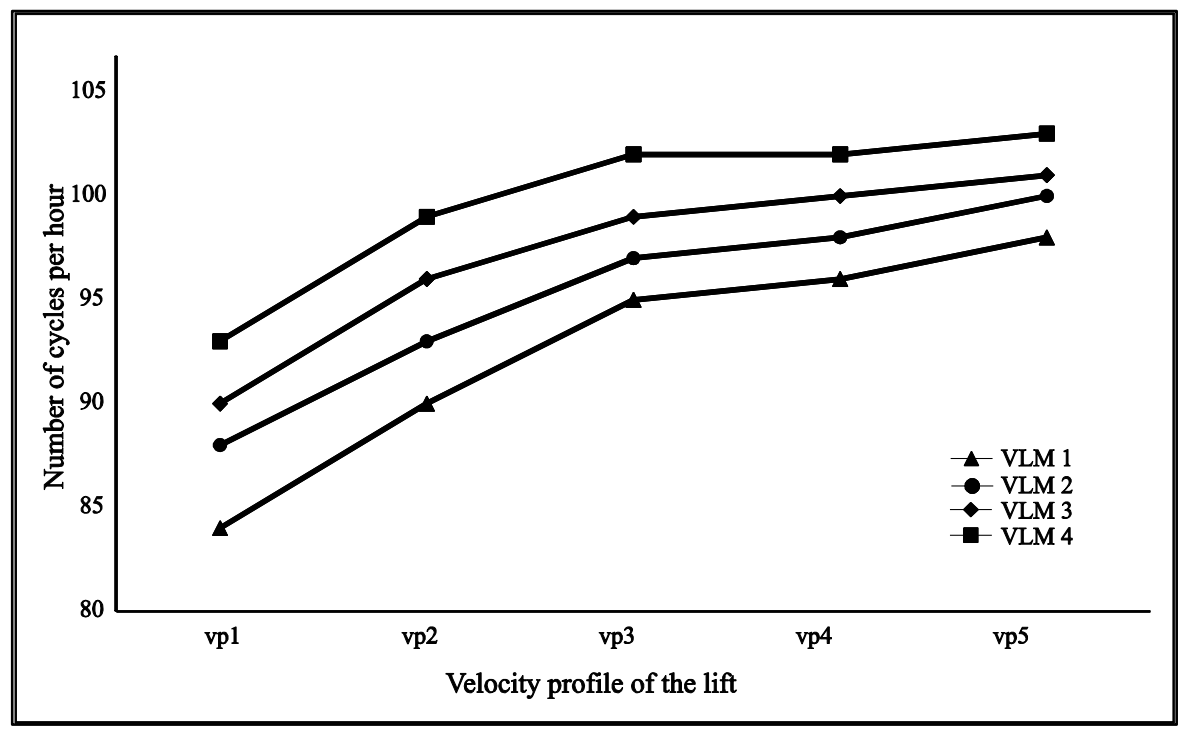

Figure 5: VLM performance in number of trays per hour when $t_{\text {pick }}=15 \mathrm{sec}$.

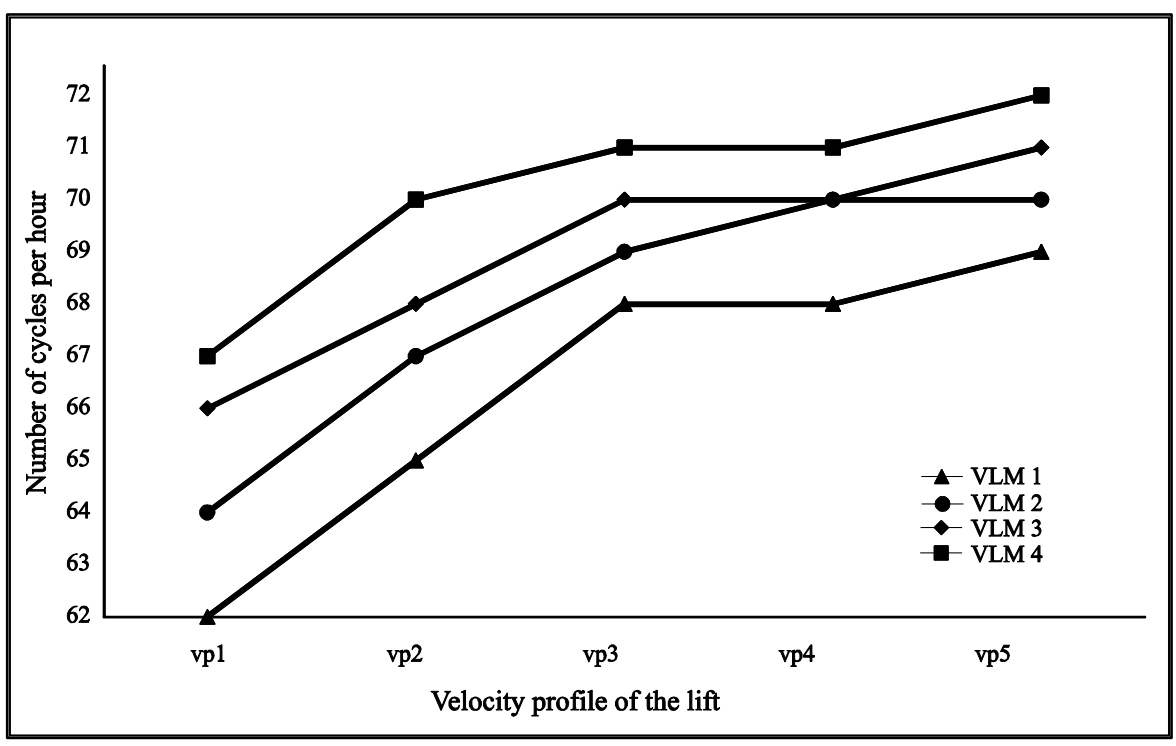

Figure 6: VLM performance in number of trays per hour when $t_{\text {pick }}=30 \mathrm{sec}$. 
For the reason that the throughput performance $\lambda(R)$ is inversly dependent on the mean cycle (retrieval) time $T(R)$, the highest throughput performance $\lambda(R)$ belongs to the VLM with small heights $H$ of the VLM $\left(\mathrm{VLM}_{4}\right)$ and better velocity profile $\mathrm{vp}_{i}$ of the lift $\left(\mathrm{vp}_{5}\right)$. Opposite, the lowest throughput performance $\lambda(R)$ belongs to the VLM with relatively large heights $H$ of the VLM $\left(\mathrm{VLM}_{1}\right)$ and moderate velocity profile $\mathrm{vp}_{i}$ of the lift $\left(\mathrm{vp}_{1}\right)$.

According to picking times (5, 15 and 30 seconds) of the order picker (operator) at the $\mathrm{I} / \mathrm{O}$ location, the next relationships can be noticed. It is expected that lower VLM with faster lift will achieve higher throughputs. However, the difference in throughput between alternatives also depends on picking time. For small values of picking times (in this case 5 seconds), the height $H$ of the VLM and the velocity profile $\mathrm{vp}_{i}$ of the lift show a significant influence on the throughput performance $\lambda(R)$ between different VLM configurations (Figs. 4 to 6 ). The lowest $\mathrm{VLM}_{4}$ reaches higher throughput performances of 11-16 trays per hour (8-15\% increased throughput) compared to the highest $\mathrm{VLM}_{1}$, depending on the velocity profile. On the contrary, for higher values of picking times (in this case 30 seconds), the height $H$ of the VLM and the velocity profile $\mathrm{vp}_{i}$ of the lift show smaller influence on the throughput performance $\lambda(R)$ between different VLM configurations (Figs. 4 to 6). In this case, the lowest $\mathrm{VLM}_{4}$ reaches higher throughput performances of only 3-5 trays per hour (4-8\% increased throughput) compared to the highest $\mathrm{VLM}_{1}$, depending on the velocity profile. If one is to choose between all alternatives (heights and velocity profiles), the lowest and fastest $\mathrm{VLM}_{4}$ with $\mathrm{vp}_{5}$ will achieve $32 \%$ better throughput compared to the highest and slowest $\mathrm{VLM}_{1}$ with $\mathrm{vp}_{1}$ in case of the picking time of 5 seconds, while just $16 \%$ in case of the picking time of 30 seconds.

\section{CONCLUSION}

This paper presents VLM's performance analysis using simulation. The simulation model of the VLM is based on the real velocity-time dependence for the lift in the vertical direction, assuming randomized storage assignment.

Various VLMs have been examined alternating VLM design (height and width) and the velocity of the lift in order to investigate the throughput performance of the VLM. Main result of this study is observation that the throughput performance $\lambda(R)$ of the VLM significantly depends on the small heights $H$ of the VLM $\left(\mathrm{VLM}_{4}\right)$ and better velocity profile $\mathrm{vp}_{i}$ of the lift $\left(\mathrm{vp}_{5}\right)$. Hence, if we decrease the height $H$ of the VLM and use even better velocity profile $\mathrm{vp}_{i}$ of the lift, we can obtain better throughput capacity (however smaller VLMs for required storage capacity comes with the increased floor space of the VLM).

Generally, when deciding on a VLM design, picking time, height $H$ of the VLM and the velocity profile $\mathrm{vp}_{i}$ of the lift have significant influance on system's performance. For small values of picking times, the height $H$ of the VLM and the velocity profile $\mathrm{vp}_{i}$ of the lift show an expressive influence on the throughput performance $\lambda(R)$ between different VLM configurations. On the contrary, for high values of picking times, the height $H$ of the VLM and the velocity profile $\mathrm{vp}_{i}$ of the lift show relatively small influence on the throughput performance $\lambda(R)$ between different VLM configurations.

According to the proposed simulation model for the design of VLM, we can conclude, that presented results are useful for engineering practice. Based on the results shown in Figs. 4, 5 and 6 and in Table III, one can relatively quickly select the most efficient type of VLM.

\section{REFERENCES}

[1] Rosi, B.; Mulej, M. (2005). Dialectical network thinking - a new system theory, Proceedings of the WOSC $13^{\text {th }}$ International Congress of Cybernetics and Systems, Vol. 6, 111-120 
[2] Rosi, B. (2015). Innovation in systems thinking: the application of dialectical network thinking in resolving complex problems, Nova Science Publishers, New York

[3] Roodbergen, K. J. (2001). Layout and routing methods for warehouses, Ph.D. dissertation, Delft University of Technology, Erasmus University Rotterdam

[4] Bartholdi, J. J.; Hackman, S. T. (2014). Warehouse \& distribution science, Release 0.96, The Supply Chain and Logistics Institute, School of Industrial and Systems Engineering, Georgia Institute of Technology, Atlanta

[5] Heragu, S. S.; Du, L.; Mantel, R. J.; Schuur, P. C. (2005). Mathematical model for warehouse design and product allocation, International Journal of Production Research, Vol. 43, No. 2, 327-338, doi: $10.1080 / 00207540412331285841$

[6] Hausman, W. H.; Schwarz, L. B.; Graves, S. C. (1976). Optimal storage assignment in automatic warehousing systems, Management Science, Vol. 22, No. 6, 629-638, doi:10.1287/mnsc.22.6.629

[7] Graves, S. C.; Hausman, W. H.; Schwarz, L. B. (1977). Storage-retrieval interleaving in automatic warehousing systems, Management science, Vol. 23, No. 9, 935-945, doi: $10.1287 / \mathrm{mnsc} .23 .9 .935$

[8] Gudehus, T. (1973). Principles of order picking: Operations in distribution and warehousing systems, W. Girardet Verlag, Essen

[9] Bozer, Y. A.; White, J. A. (1984). Travel-time models for automated storage/retrieval systems, IIE Transactions, Vol. 16, No. 4, 329-338, doi:10.1080/07408178408975252

[10] Hwang, H.; Lee, S. B. (1990). Travel-time models considering the operating characteristics of the storage and retrieval machine, International Journal of Production Research, Vol. 28, No. 10, 1779-1789, doi:10.1080/00207549008942833

[11] Sari, Z.; Saygin, C.; Ghouali, N. (2005). Travel-time models for flow-rack automated storage and retrieval systems, International Journal of Advanced Manufacturing Technology, Vol. 25, No. 910, 979-987, doi:10.1007/s00170-003-1932-3

[12] Lerher, T.; Sraml, M.; Kramberger, J.; Potrc, I.; Borovinsek, M.; Zmazek, B. (2005). Analytical travel time models for multi-aisle automated storage and retrieval systems, International Journal of Advanced Manufacturing Technology, Vol. 30, No. 3-4, 340-356, doi:10.1007/s00170-005$\underline{0061-6}$

[13] Gu, J.-X.; Goetschalckx, M.; McGinnis, L. F. (2007). Research on warehouse operation: a comprehensive review, European Journal of Operational Research, Vol. 177, No. 1, 1-21, doi:10.1016/j.ejor.2006.02.025

[14] Roodbergen, K. J.; Vis, I. F. A. (2009). A survey of literature on automated storage and retrieval systems, European Journal of Operational Research, Vol. 194, No. 2, 343-362, doi:10.1016/ j.ejor.2008.01.038

[15] Lerher, T.; Sraml, M.; Potrc, I. (2011). Simulation analysis of mini-load multi-shuttle automated storage and retrieval systems, International Journal of Advanced Manufacturing Technology, Vol. 54, No. 1-4, 337-348, doi:10.1007/s00170-010-2916-8

[16] Lerher, T.; Ekren, Y. B.; Sari, Z.; Rosi, B. (2015). Simulation analysis of shuttle based storage and retrieval systems, International Journal of Simulation Modelling, Vol. 14, No. 1, 48-59, doi:10.2507/IJSIMM14(1)5.281

[17] Smew, W.; Young, P.; Geraghty, J. (2013). Supply chain analysis using simulation, Gaussian process modelling and optimisation, International Journal of Simulation Modelling, Vol. 12, No. 3, 178-189, doi:10.2507/IJSIMM12(3)4.239

[18] Bekker, J. (2013). Multi-objective buffer space allocation with the cross-entropy method, International Journal of Simulation Modelling, Vol. 12, No. 1, 50-61, doi:10.2507/ IJSIMM12(1)5.228

[19] Berlec, T.; Kusar, J.; Zerovnik, J.; Starbek, M. (2014). Optimization of a product batch quantity, Strojniski vestnik - Journal of Mechanical Engineering, Vol. 60, No. 1, 35-42, doi:10.5545/svjme.2013.1009

[20] Erkan, T. E.; Can, G. F. (2014). Selecting the best warehouse data collecting system by using AHP and FAHP methods, Technical Gazette, Vol. 21, No. 1, 87-93

[21] Seebacher, G.; Winkler, H.; Oberegger, B. (2015). In-plant logistics efficiency valuation using discrete event simulation, International Journal of Simulation Modelling, Vol. 14, No. 1, 60-70, doi:10.2507/IJSIMM14(1)6.289 
[22] Karabegovic, I.; Karabegovic, E.; Mahmic, M.; Husak, E. (2015). The application of service robots for logistics in manufacturing processes, Advances in Production Engineering \& Management, Vol. 10, No. 4, 185-194, doi:10.14743/apem2015.4.201

[23] Meller, R. D.; Klote, J. F. (2004). A throughput model for carousel/VLM pods, IIE Transactions, Vol. 36, No. 8, 725-741, doi:10.1080/07408170490458472

[24] Dukic, G.; Opetuk, T.; Lerher, T. (2015). A throughput model for a dual-tray Vertical Lift Module with a human order-picker, International Journal of Production Economics, Vol. 170, Part C, 874-881, doi:10.1016/j.ijpe.2015.04.009 